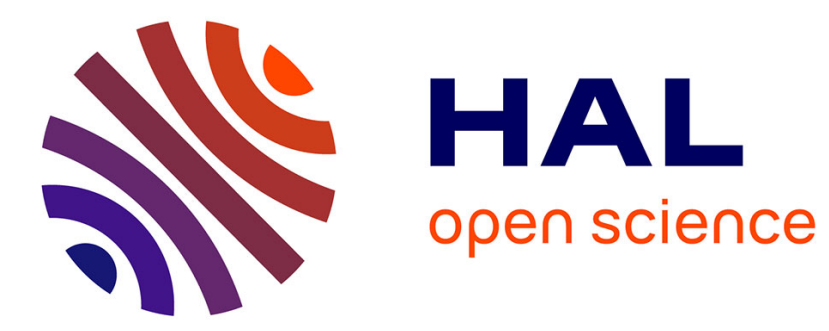

\title{
Spin-glass magnetism of the non-equiatomic CoCrFeMnNi high-entropy alloy
}

\author{
P. Koželj, S. Vrtnik, M. Krnel, A. Jelen, D. Gačnik, M. Wencka, Z. Jagličić,
}

A. Meden, G. Dražić, Frederic Danoix, et al.

\section{> To cite this version:}

P. Koželj, S. Vrtnik, M. Krnel, A. Jelen, D. Gačnik, et al.. Spin-glass magnetism of the non-equiatomic CoCrFeMnNi high-entropy alloy. Journal of Magnetism and Magnetic Materials, 2021, 523, pp.167579. 10.1016/j.jmmm.2020.167579 . hal-03365254

\section{HAL Id: hal-03365254 https://hal.science/hal-03365254}

Submitted on 5 Oct 2021

HAL is a multi-disciplinary open access archive for the deposit and dissemination of scientific research documents, whether they are published or not. The documents may come from teaching and research institutions in France or abroad, or from public or private research centers.
L'archive ouverte pluridisciplinaire HAL, est destinée au dépôt et à la diffusion de documents scientifiques de niveau recherche, publiés ou non, émanant des établissements d'enseignement et de recherche français ou étrangers, des laboratoires publics ou privés. 


\title{
Spin-glass magnetism of the non-equiatomic CoCrFeMnNi high-entropy alloy
}

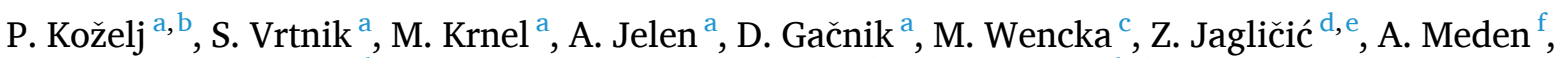

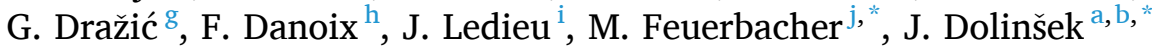 \\ ${ }^{a}$ Jožef Stefan Institute, Jamova 39, SI-1000 Ljubljana, Slovenia \\ ${ }^{\mathrm{b}}$ University of Ljubljana, Faculty of Mathematics and Physics, Jadranska 19, SI-1000 Ljubljana, Slovenia \\ ${ }^{\mathrm{c}}$ Institute of Molecular Physics, Polish Academy of Sciences, Smoluchowskiego 17, PL-60-179 Poznań, Poland \\ ${ }^{\mathrm{d}}$ Institute of Mathematics, Physics and Mechanics, Jadranska 19, SI-1000 Ljubljana, Slovenia \\ e University of Ljubljana, Faculty of Civil and Geodetic Engineering, Jamova 2, SI-1000 Ljubljana, Slovenia \\ ${ }^{\mathrm{f}}$ University of Ljubljana, Faculty of Chemistry and Chemical Technology, Večna pot 113, SI-1000 Ljubljana, Slovenia \\ ${ }^{g}$ National Institute of Chemistry, Department of Materials Chemistry, Hajdrihova 19, SI-1000 Ljubljana, Slovenia \\ ${ }^{\mathrm{h}}$ Normandie Université, UNIROUEN, INSA Rouen, CNRS, Groupe de Physique des Matériaux, 76000 Rouen, France \\ i Institut Jean Lamour, UMR 7198 CNRS - Université de Lorraine, Campus Artem, 2 allée André Guinier, BP 50840, 54011 Nancy Cedex, France \\ ${ }^{\mathrm{j}}$ Institut für Mikrostrukturforschung, Forschungszentrum Jülich, D-52425 Jülich, Germany
}

\section{A R T I C L E I N F O}

\section{Keywords:}

High-entropy alloys

Spin glasses

\begin{abstract}
A B S T R A C T
The CoCrFeMnNi high-entropy alloy (HEA) is a magnetically concentrated crystalline system with all lattice sites magnetic, containing randomness (five different types of spins are randomly positioned on the lattice) and frustration (a consequence of mixed ferromagnetic and antiferromagnetic interactions). The sample material was prepared as a non-equiatomic, fully random solid solution of the five magnetic elements and we have studied experimentally the nature of the magnetic state. Upon cooling, no long-range magnetic ordering takes place, but the spin system undergoes a kinetic freezing transition to a spin glass phase, where below the spin freezing temperature $T_{f} \approx 20 \mathrm{~K}$, ergodicity of the system is broken. The observed broken-ergodicity phenomena include zero-field-cooled - field-cooled magnetization splitting in low magnetic fields, a frequency-dependent cusp in the ac susceptibility, an ultraslow time-decay of the thermoremanent magnetization and the memory effect, where the state of the spin system reached upon isothermal aging at a certain temperature can be retrieved after a reverse temperature cycle. All these phenomena are associated with the out-of-equilibrium dynamics of a nonergodic, frustrated system of coupled spins that approach thermal equilibrium, but can never reach it on a finite experimental time scale, so that we are observing only transient effects of partial equilibration within localized spin domains.
\end{abstract}

\section{Introduction}

The low-temperature magnetic state of the quinary face-centered cubic (fcc) CoCrFeMnNi high-entropy alloy (HEA) remains an open issue. This alloy is composed of five $3 d$ magnetic elements, where $\mathrm{Fe}$, Co and $\mathrm{Ni}$ are ferromagnetic (FM) as elemental metals, whereas $\mathrm{Mn}$ and $\mathrm{Cr}$ are antiferromagnetic (AFM). Ab initio calculations have predicted that the Fe, Co and Ni magnetic moments favor parallel (FM) alignment, the $\mathrm{Ni}$ moments are vanishing small, and the $\mathrm{Cr}$ and $\mathrm{Mn}$ moments orient antiparallel (AFM) to the moments of $\mathrm{Fe}$ and $\mathrm{Co}$ [1-4]. In another theoretical study [5], the Ni paramagnetic moments were predicted to be nonzero, albeit smaller than the moments of the other four elements. It was reported that the CoCrFeMnNi HEA undergoes two successive phase transitions, where the higher transition at $93 \mathrm{~K}$ is paramagnetic to spin glass, and the lower transition at $38 \mathrm{~K}$ is FM [1]. In another report [6], the temperature-dependent field-cooled magnetization data indicate just one transition, occurring at about $25 \mathrm{~K}$ to a spin glass state, but no additional evidence was given to support this claim.

The CoCrFeMnNi HEA belongs to the class of "regular" solid solutions with nonzero mixing enthalpy, $\Delta H_{\text {mix }} \neq 0$, and slightly different

\footnotetext{
* Corresponding authors at: Institut für Mikrostrukturforschung, Forschungszentrum Jülich, D-52425 Jülich, Germany (M. Feuerbacher) and Jožef Stefan Institute, Jamova 39, SI-1000 Ljubljana, Slovenia (J. Dolinšek).

E-mail addresses: m.feuerbacher@fz-juelich.de (M. Feuerbacher), janez.dolinsek@ijs.si (J. Dolinšek).
} 
(though very similar) atomic radii of the elements, where both effects introduce local lattice distortions. The alloy can decompose into multiple phases when annealed at temperatures below about $1073 \mathrm{~K}$, but it can also be prepared as a single-phase fcc material with close to random distribution of the elements [7-9]. Such a chemically disordered and topologically distorted lattice entails a broad distribution of nearestneighbor chemical environments and bond lengths for a given magnetic atom, leading to distributions of site moments $P(\mu)$, exchange interactions $P(\mathscr{f})$, magnetic anisotropy $P(D)$ and dipolar magnetic fields $P\left(H_{d}\right)$. In a metallic environment, the $3 d$ moments couple indirectly via the conduction electrons, leading to the long-range oscillatory Ruderman-Kittel-Kasuya-Yosida (RKKY) coupling between the moments, where the sign of the coupling fluctuates between a positive and a negative value on the scale of nanometers. Combined with the random distribution of five magnetic elements on the lattice, with a preference for either parallel (Co, Fe, Ni) or antiparallel ( $\mathrm{Cr}, \mathrm{Mn}$ ) nearest-neighbor spin alignment, the spin system assumes a frustrated configuration. The CoCrFeMnNi HEA represents a magnetically concentrated system with all lattice sites magnetic, containing randomness (five different types of spins are placed randomly on the lattice) and frustration (no spin configuration can satisfy all the bonds and minimize the energy at the same time). Randomness and frustration are the two necessary ingredients to classify a given spin system into the broad class of spin glasses [10]. This class contains a wide range of magnetically diluted and concentrated materials, crystalline or amorphous, site-disordered or site-ordered, but geometrically frustrated. The cooperative magnetism of such a system is highly complex. In this paper we study the nature of the magnetic state in the non-equiatomic CoCrFeMnNi HEA, with the aim to find out whether the spin system undergoes any long-range magnetic ordering (FM or AFM) upon cooling, or the $T \rightarrow 0$ ground state is a spin glass.

\section{Material synthesis and characterization}

The sample material was grown by the Czochralski technique and the details are given in the Experimental section. Laue inspection revealed that it contained several grains with diameters between about 1 and 3 $\mathrm{mm}$. The XRD pattern of the employed CoCrFeMnNi material is shown in Fig. 1, confirming its single-phase fcc structure (space group $\mathrm{Fm}^{-} \mathrm{m}$ ), with the unit cell parameter $a=0.358 \mathrm{~nm}$. The XRD pattern shows the (200) and (111) reflections only (a trace of the (311) reflection can also be noticed), revealing strong orientational ordering of the coherently scattering domains and indicating that the material was close to a single crystal, due to the millimeter-size grains.

The microstructure was studied by SEM backscattered electron (BSE) imaging and EDS elemental mapping (Fig. 2) on a cross section of the surface prepared by $\mathrm{Ga}$ ion beam in an FIB-SEM instrument. A

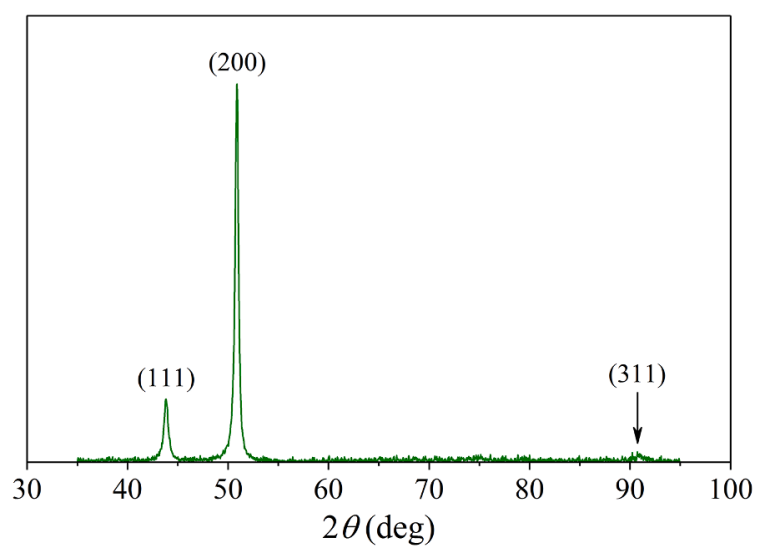

Fig. 1. XRD pattern of the CoCrFeMnNi HEA at room temperature, using $\mathrm{Cu}$ $\mathrm{K} \alpha_{1}$ radiation $(\lambda=1.54056 \AA)$. The peaks are indexed to the fcc crystal lattice. homogeneous microstructure was found, with a random distribution of the five elements on the micrometer scale. The average composition determined by SEM EDS, $\mathrm{Co}_{23.7} \mathrm{Cr}_{23.2} \mathrm{Fe}_{24.5} \mathrm{Mn}_{7.7} \mathrm{Ni}_{20.9}$, was nonequiatomic due to Mn evaporation from the melt.

Atom probe tomography (APT) was used to analyze the local chemical composition and check for possible local inhomogeneities on the nanometric scale. The details are given in the Experimental section. Average compositions were calculated using the background correction option, and isobars were corrected according to the natural isotopic ratios. The APT-determined average composition of the material was $\mathrm{Co}_{24.2} \mathrm{Cr}_{21.4} \mathrm{Fe}_{25.5} \mathrm{Mn}_{7.2} \mathrm{Ni}_{21.7}$, in agreement with the one determined by SEM EDS (within the experimental precision of about 1 at. \%). The (APT) 3D reconstructions of the elements are shown in Fig. 3a. The reconstructions reveal a homogeneous, random elemental distribution on the nanoscale, with no visible compositional heterogeneities. In order to investigate short range atomic ordering, the distances between the first nearest neighbors were plotted, as shown in Fig. 3b. To see whether FM or AFM atomic clustering is present at the nanometric scale, the FM elements $\mathrm{Fe}$, $\mathrm{Co}$ and $\mathrm{Ni}$ and the AFM elements $\mathrm{Cr}$ and $\mathrm{Mn}$ were considered separately, by calculating the first-nearest-neighbor (FNN) distances between the elements of the same magnetic kind (FM or AFM). Here we note that these are the distances in the $3 \mathrm{D}$ reconstructions, where atoms are positioned with a lateral (as referred to the specimen axis) accuracy of about $0.3 \mathrm{~nm}$, due to the spatial resolution of the instrument [11]. This is the reason why the FNN distances smaller than the minimum theoretical one (which, for the fcc structure with the lattice parameter $a=0.358 \mathrm{~nm}$ amounts to $a / \sqrt{2}=0.25 \AA$ ) are experimentally observed. The height of each distribution is proportional to the amount of the FM and AFM atoms. The experimental distributions (solid symbols) were then compared to the ones corresponding to the "randomized" configuration. Such a configuration is obtained by randomly positioning all five types of atoms on the experimental lattice sites, in order to mimic a perfectly random distribution, and the FNN distances between the FM and the AFM atoms recalculated for this configuration. For the same reason as for the experimental distributions, FNN distances smaller than the minimum theoretical one are again observed. The randomized distributions (dashed and solid curves) are identical to the experimental ones, revealing that the distribution of the five different elements within the APT-investigated volume was homogeneous, with no precipitation or segregation observed.

The possible existence of short-range chemical ordering on the scale of nearest neighbors was also investigated by high-angle annular dark field (HAADF) scanning transmission electron microscope (STEM) imaging and EDS elemental mapping. The details are given in the Experimental section. The HAADF STEM image (Fig. 4a) reveals some intensity fluctuations on the nanometric scale, but these are so small that they could originate either from the chemical composition difference or from thickness variations. The expected difference of intensities in the $Z$ contrast imaging due to eventual chemical composition fluctuations in the CoCrFeMnNi HEA is small since the atomic numbers of the elements are so similar (the elements follow each other in the periodic system from $\mathrm{Cr}(Z=24)$ to $\mathrm{Ni}(Z=28)$, making the total difference in $Z$ of about $15 \%$ only). In order to unravel the origin of the intensity fluctuations, we have performed EELS spectroscopy of two nearby areas of about $2 \mathrm{~nm}$ cross dimension, one bright and the other dark (shown encircled in Fig. 4a), separated by a few nm distance. For the areas spaced so closely, it is reasonable to assume that the thickness of the sample does not change. This was confirmed by estimating the sample thickness from the low-energy EELS spectra, where the thickness difference of the two regions was found within $10 \%$, so that the intensity variations are due to the composition difference. The superimposed EELS spectra of the bright and dark regions, normalized to the $\mathrm{Fe}_{3}$ intensity are shown in Fig. $4 \mathrm{~b}$. The most significant difference is a small increase of the $\mathrm{Ni}$ concentration in the bright region relative to the dark region (the $\mathrm{Ni}_{3}$ lines are shown expanded in the inset). The local 

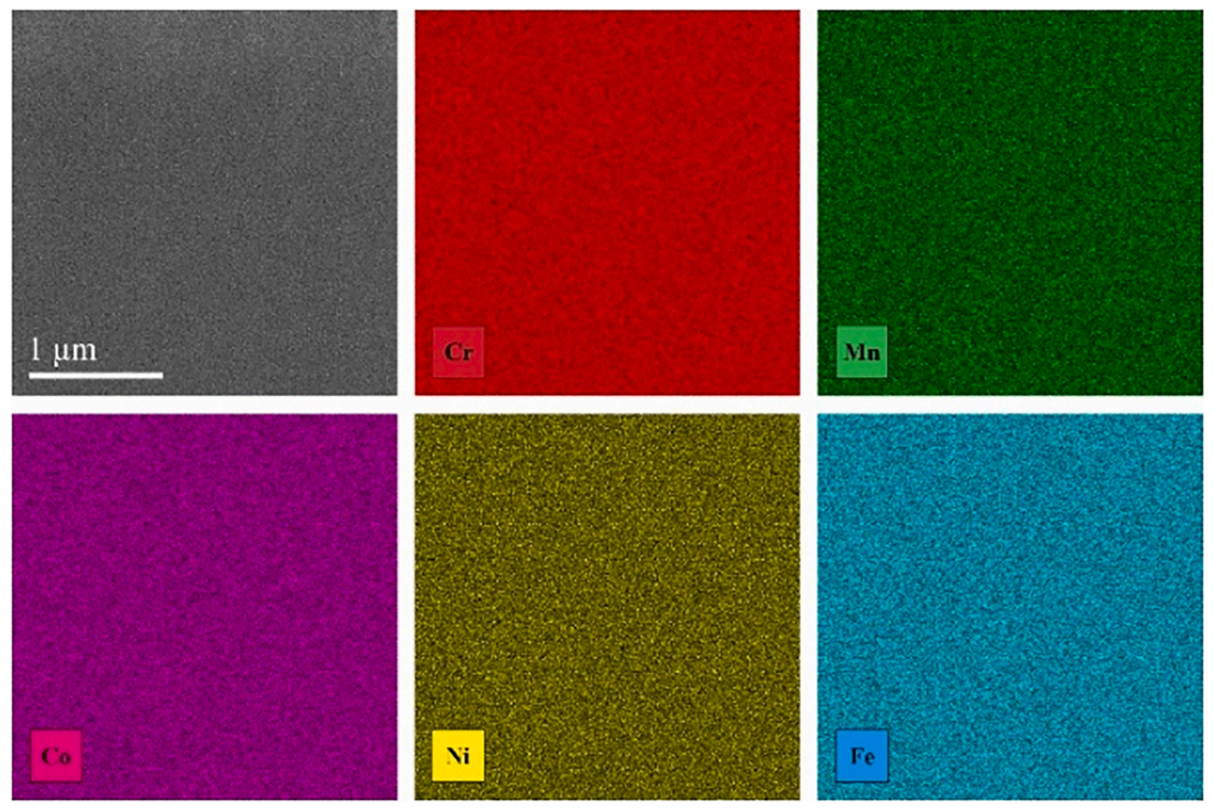

Fig. 2. SEM BSE image (upper left corner) and EDS elemental maps of the CoCrFeMnNi HEA on the micrometer scale.

a)
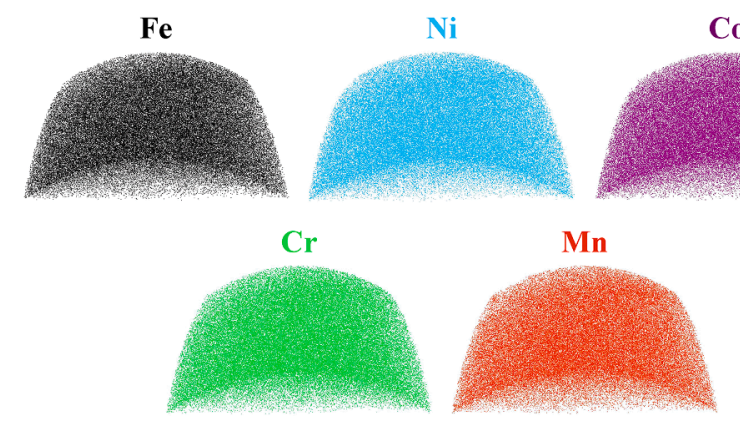

$20 \mathrm{~nm}$

b)

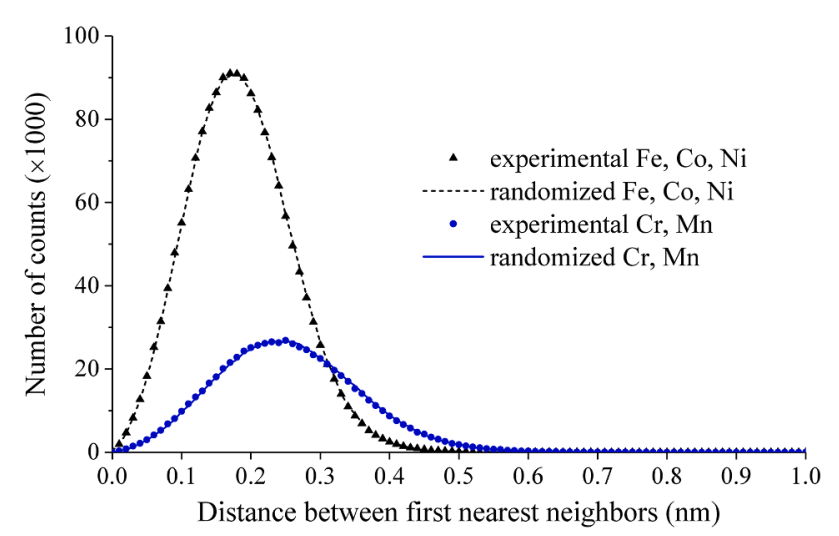

Fig. 3. (a) APT 3D reconstructions of the elements. (b) First-nearest-neighbor distances between the elements of the same magnetic kind (FM elements Fe, $\mathrm{Co}$, Ni or AFM elements $\mathrm{Cr}, \mathrm{Mn}$ ), obtained from the 3D reconstructions. The experimental distributions are compared to the corresponding theoretical "randomized" configurations.

compositions were evaluated quantitatively by STEM EDS. The composition of the bright area was found to be $\mathrm{Co}_{23.9} \mathrm{Cr}_{20.3}$ $\mathrm{Fe}_{24.0} \mathrm{Mn}_{7.7} \mathrm{Ni}_{24.1}$, whereas the composition of the dark area was $\mathrm{Co}_{25.1} \mathrm{Cr}_{22.4} \mathrm{Fe}_{23.6} \mathrm{Mn}_{7.5} \mathrm{Ni}_{21.4}$. The main differences are a small enrichment of the Ni concentration (by about $+3 \%$ ) and depletion of the $\mathrm{Cr}$ concentration (by $-2 \%$ ) in the bright region relative to the dark one (both differences are statistically relevant). The size of the areas with a different chemical composition is about $2 \mathrm{~nm}$. Since the RKKY exchange interaction responsible for the magnetic ordering is a long-range interaction, extending over many shells of interacting spins, such small-scale composition fluctuations have negligible effect on the magnetism of the material and the distribution of different spin species on the lattice can be considered as random. In other words, the investigated CoCrFeMnNi HEA material is very close to a fully random solid solution.

To estimate the size of the domains with a different chemical composition, a geometric phase analysis (GPA) was also performed. GPA is a TEM method for measuring and mapping the displacements and strain fields. In the CoCrFeMnNi HEA, the strains are introduced by slightly different sizes of the unit cells. Fig. 4c shows maps of the $\varepsilon_{x x}, \varepsilon_{y y}$ and $\varepsilon_{x y}$ strain-tensor components over the area of $8 \times 8 \mathrm{~nm}^{2}$, where interfaces between the domains are clearly visible. The GPA analysis confirms that the cross dimensions of the domains with slightly different composition are about $2 \mathrm{~nm}$.

\section{Results}

\subsection{Temperature-dependent $d c$ and ac magnetization and susceptibility}

The zero-field-cooled (zfc) and field-cooled (fc) direct-current (dc) magnetization $M$ in a magnetic field $\mu_{0} H=10 \mathrm{mT}$ is shown in Fig. $5 \mathrm{a}(M$ is presented in Bohr magnetons $\mu_{B}$ per formula unit (f.u.), i.e., per one $\mathrm{Co}_{0.237} \mathrm{Cr}_{0.232} \mathrm{Fe}_{0.245} \mathrm{Mn}_{0.077} \mathrm{Ni}_{0.209}$ "average" atom of the structure). The $M(T)$ curve shows a paramagnetic-like growth upon cooling down to about $20 \mathrm{~K}$. Below that temperature, the zfc and fc magnetizations $M_{z f c}$ and $M_{f c}$ start to differ, where $M_{f c}$ stays approximately constant upon further cooling, whereas $M_{z f c}$ shows a cusp and then decreases towards zero. A large $M_{z f c}-M_{f c}$ difference in a low magnetic field is typical of a frustrated spin system with a broken ergodicity and the temperature, where the $\mathrm{zfc}-\mathrm{fc}$ splitting sets in, is associated with the spin freezing temperature $T_{f}$.

The magnetization data from Fig. 5a, converted to the susceptibility $\chi=M / H$, were analyzed in the paramagnetic regime $\left(T>T_{f}\right)$ with the Curie-Weiss law, $\chi=C /(T-\theta)$. The $\chi^{-1}$ versus $T$ data are shown in Fig. 5b. The diamagnetic core susceptibility, estimated from literature 

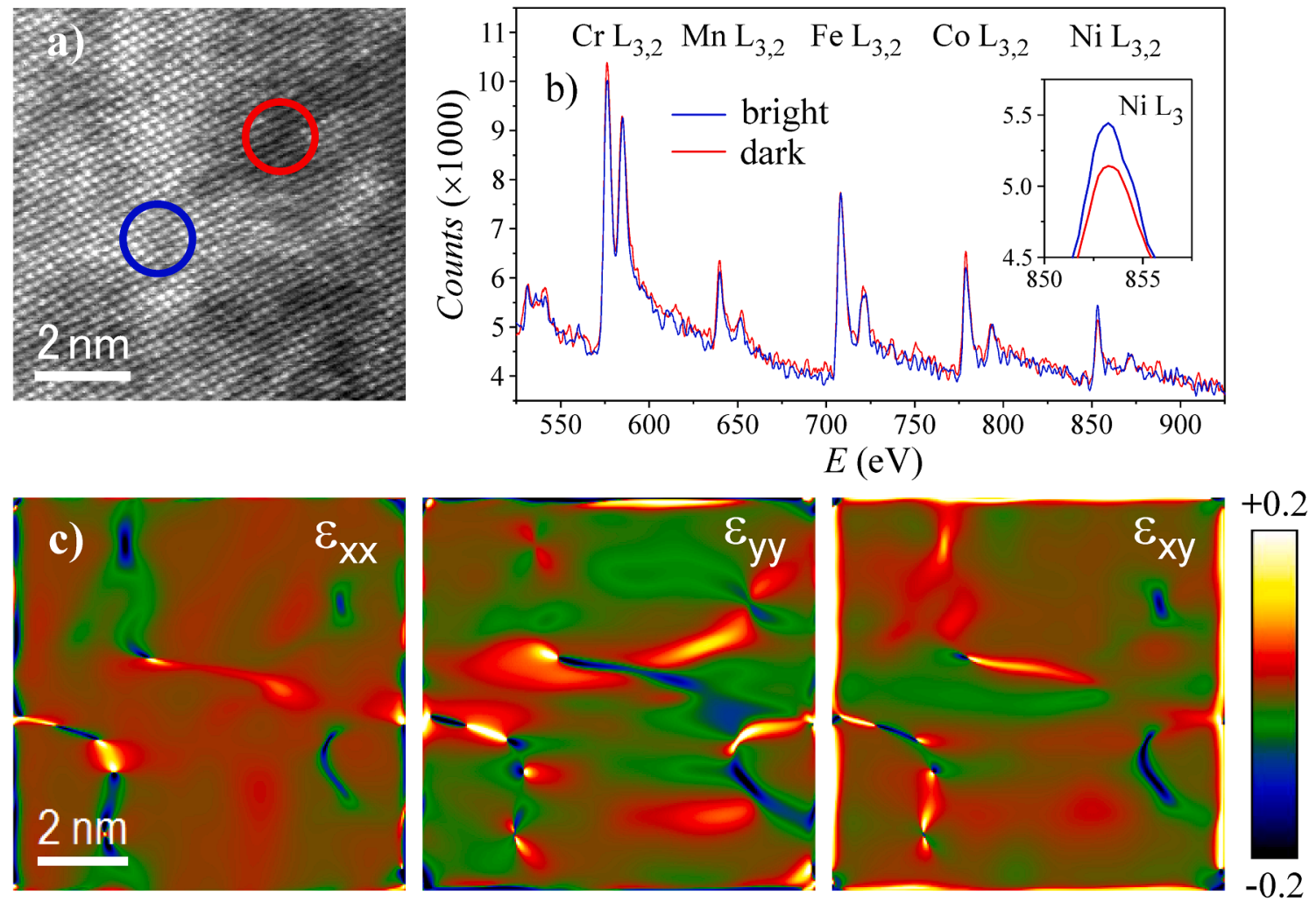

Fig. 4. (a) A HAADF STEM image of the CoCrFeMnNi material on the atomic-resolution scale. Blue and red circles surround a bright and a dark region, respectively, of slightly different chemical composition. (b) Superimposed EELS spectra of the bright (blue trace) and dark (red trace) regions, normalized to the Fe $\mathrm{L}_{3}$ intensity (the inset shows $\mathrm{Ni} \mathrm{L}_{3}$ lines on an expanded scale). (c) Maps of the $\varepsilon_{x x}$, $\varepsilon_{y y}$ and $\varepsilon_{x y}$ strain tensor components. (For interpretation of the references to colour in this figure legend, the reader is referred to the web version of this article.)

tables, is negligible and has not been subtracted. The fit in the paramagnetic regime (solid line in Fig. 5b) yielded the Curie-Weiss constant $C=6.6 \times 10^{-6} \mathrm{~m}^{3} \mathrm{~K} / \mathrm{mol}$. The mean effective magnetic moment $\mu_{\text {eff }}^{-}=$ $p_{\text {eff }}^{-} \mu_{B}$ (with $p_{\text {eff }}^{-}$denoting the mean effective Bohr magneton number) was determined from the formula $p_{\text {eff }}^{-}=\left(797.7 \sqrt{\mathrm{mol} / \mathrm{m}^{3} \mathrm{~K}}\right) \sqrt{\mathrm{C}}[12]$, yielding $\mu_{\text {eff }}^{-}=(2.1 \pm 0.1) \mu_{B}$. The Curie-Weiss temperature was determined to be $\theta=19 \mathrm{~K}$, where its positive value indicates that the FMtype interactions in the material dominate over the AFM-type ones.

The real part of the alternating-current (ac) susceptibility $\chi$, measured in a magnetic field of amplitude $\mu_{0} H_{0}=0.8 \mathrm{mT}$ and logarithmically spaced frequencies $\nu$ between 1 and $1000 \mathrm{~Hz}$, is presented in Fig. 6a. A frequency-dependent peak is observed at about $21 \mathrm{~K}$, which shifts to higher temperatures at higher frequencies (shown on an expanded scale in Fig. 6b) that is indicative of a spin freezing transition to a nonergodic state. The temperature of the $\chi$ ' maximum is conveniently defined as the frequency-dependent spin freezing temperature $T_{f}(\nu)$. The normalized spin freezing temperature $T_{f}(\nu) / T_{f}(1 \mathrm{~Hz})$ is presented in the inset of Fig. $6 \mathrm{~b}$. The fractional shift of the spin freezing temperature per decade of frequency was evaluated as $\Gamma=\Delta T_{f}$ / $T_{f} \Delta(\log \nu)=1.2 \times 10^{-2}$, a value typically encountered in spin glasses [13].

\section{2. $M(H)$ curves}

The magnetization versus the magnetic field curves, $M(H)$, at selected temperatures between 25 and $2 \mathrm{~K}$ are displayed in Fig. 7a. A hysteresis starts to develop at temperatures below $10 \mathrm{~K}$, with the coercive field at $T=2 \mathrm{~K}$ amounting to $\mu_{0} H_{c}=0.13 \mathrm{~T}$. At large fields, the $M(H)$ curves saturate to an inclined line.

By considering theoretically the shape of the $M(H)$ curves, we as- sume that the CoCrFeMnNi HEA is an exchange-dominated system. It is well established that in chemically disordered $3 d$ magnetic alloys, random local magnetic anisotropy at the atomic scale is generally insufficient to pin the local magnetization direction. The type of magnetic ordering is then essentially determined by the distribution of the exchange coupling constants $P(\mathscr{f})$. Since the CoCrFeMnNi HEA is a system with mixed FM/AFM interactions because of close to random mixing of the FM elements $\mathrm{Co}$, Fe and $\mathrm{Ni}$ with the AFM elements $\mathrm{Cr}$ and $\mathrm{Mn}$, it is straightforward to assume that there exist nanometer-scale spin domains with a predominant FM coupling and spin domains with a predominant AFM coupling. The $P(\mathscr{f})$ distribution can be assumed a continuous and symmetric distribution function that extends on both $\mathscr{J}>0$ (FM coupling) and $\mathscr{J}<0$ (AFM coupling) sides of the $\mathscr{J}$ axis [14]. A spin glass $P(\mathscr{f})$ is, by definition, characterized by a zero average exchange interaction, $\mathscr{J}^{-}=0$, whereas a $P(\mathscr{J})$ biased towards a net positive value, $\mathscr{J}^{-}>0$, designates asperomagnetic spin order (a subclass of spin glasses in a broader sense, known in the context of amorphous magnets $[14,15])$. Both types of $P(\mathscr{J})$ distributions are shown schematically in Fig. $7 \mathrm{c}$.

The $M(H)$ curves were modeled by a function

$M=M_{0} L(x)+k \mu_{0} H$

The term $M_{0} L(x)$ accounts for the part of $P(\mathscr{f})$ on the $\mathscr{J}>0$ (FM) side, where $L(x)$ with $x=\mu \mu_{0} H /\left(k_{B} T\right)$ is the Langevin function. The Langevin model assumes that the magnetic moments $\mu=g \mu_{B} S$ (where $g$ is the Landé factor) are classical vectors and the spin $S$ can assume any value $(S \rightarrow \infty)$, accounting for the large effective FM group spins. For large $x$, the Langevin function saturates to a constant (horizontal) plateau. The linear term $k \mu_{0} H$ accounts for the part of $P(\mathscr{f})$ on the $\mathscr{J}<0$ (AFM) side and $\mu_{0} k$ represents the AFM susceptibility. For large $H$, the $M(H)$ curve of Eq. (1) asymptotically approaches an inclined linear line with the slope $\mu_{0} k$. This is a decisive difference to ferromagnets, which 

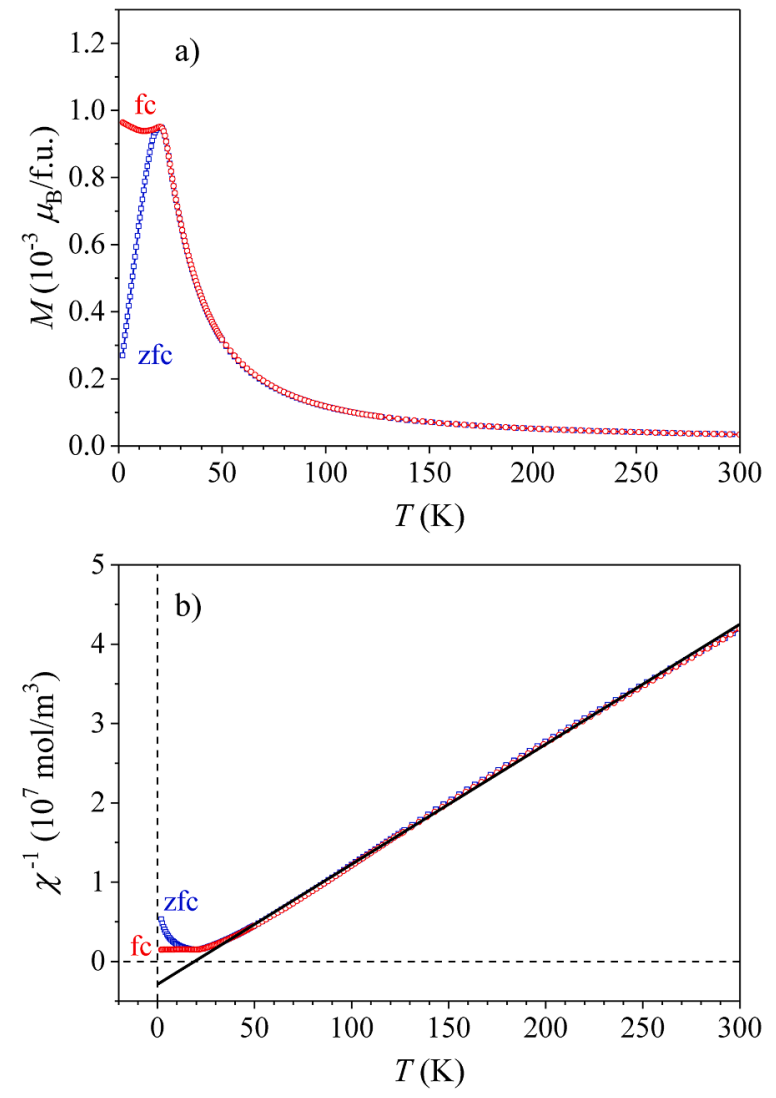

Fig. 5. (a) Temperature-dependent zfc and fc dc magnetizations between 300 and $2 \mathrm{~K}$ in a magnetic field $\mu_{0} H=10 \mathrm{mT}$. The "f.u." denotes formula unit, i.e., one $\mathrm{Co}_{0.237} \mathrm{Cr}_{0.232} \mathrm{Fe}_{0.245} \mathrm{Mn}_{0.077} \mathrm{Ni}_{0.209}$ "average atom" of the structure. (b) Magnetic susceptibility $\chi=M / H$ in a $\chi^{-1}$ vs. $T$ plot. Solid line is a Curie-Weiss fit in the paramagnetic regime.

saturate to a horizontal plateau and antiferromagnets, which are linear in $H$ in the entire field range. The Langevin function cannot reproduce hysteresis, so that Eq. (1) can be used for fitting the experimental $M(H)$ curves of Fig. $7 \mathrm{a}$ at temperatures above $10 \mathrm{~K}$.

The fit of the $M(H)$ curve at $T=10 \mathrm{~K}$ is presented in Fig. 7b, where the FM and AFM contributions to the total $M$ are also shown separately. The fit parameters are $M_{0}=4.8 \times 10^{-2} \mu_{B}$ /f.u., $\mu=56 \mu_{B}$ and $k=$ $9.3 \times 10^{-3} \mu_{B}$ /(f.u.T). The relatively small group moments of $56 \mu_{B}$ are consistent with the assumption of nanometer-size spin domains. The rapid FM-type initial growth of the magnetization at low fields due to the Langevin function and the saturation to an inclined line at high fields due to the AFM part of the magnetization are clearly observed, confirming qualitatively the shape of the $P(\mathscr{F})$ distribution shown schematically in Fig. 7c that extends on both sides $\mathscr{J}>0$ and $\mathscr{J}<0$ of the $\mathscr{J}$ axis. A "true" spin glass-type $P(\mathscr{J})$ with $\mathscr{J}^{-}=0$ can, however, not be discriminated from the asperomagnetic $P(\mathscr{J})$ with $\mathscr{J}^{-}>0$ on this basis. The positive Curie-Weiss temperature $\theta=19 \mathrm{~K}$ determined from the paramagnetic susceptibility (Fig. 5b) suggests that $P(\mathscr{f})$ could be slightly biased towards a net positive (FM) value, $\mathscr{J}^{-}>0$.

\subsection{Thermoremanent magnetization time-decay}

Magnetically frustrated spin systems like spin glasses generally exhibit a kinetic slowing-down dynamics upon cooling with a broad distribution of the spin fluctuation (reorientation) times, extending over many orders of magnitude. At the spin freezing temperature $T_{f}$, the spin fluctuations effectively freeze on the finite time scale of a given experimental observation technique, so that the ergodicity of the spin system is broken at $T<T_{f}$ because thermal fluctuations are no longer able to
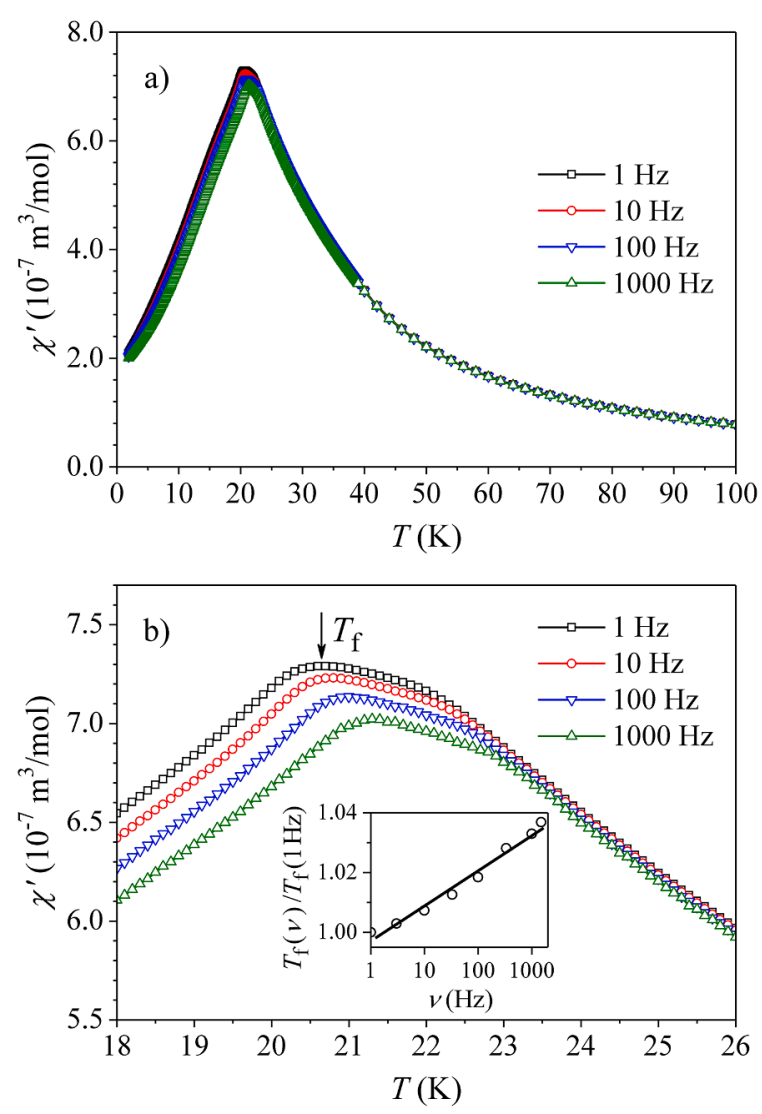

Fig. 6. (a) Real part $\chi$ ' of the ac magnetic susceptibility at frequencies $\nu=1$, 10,100 and $1000 \mathrm{~Hz}$. (b) $\chi$ ' on an expanded temperature scale around the spin freezing transition (the arrow denotes the spin freezing temperature $T_{f}$, associated with the peak of $\chi^{\prime}$ ). The inset shows the frequency-dependent spin freezing temperature $T_{f}(\nu)$, normalized to $T_{f}(1 \mathrm{~Hz})$.

maintain thermal equilibrium on the experimental time scale. What one observes is a slow approach of the spin system towards the thermodynamic equilibrium, which can never be reached due to the macroscopic equilibration times, but can still lead to a partial (local) equilibration of small spin clusters [16-22]. The logarithmically slow time-decay of the thermoremanent magnetization (TRM) is one manifestation of the outof-equilibrium dynamics of a nonergodic spin system. The TRM is measured by cooling the spin system in a field $H_{f c}$ from the paramagnetic phase through the spin freezing temperature $T_{f}$ into the nonergodic phase to a "measuring" (aging) temperature $T_{m}$, where the cooling is stopped for a "waiting" (aging) time $t_{w}$ and the spin system is let to age in the field isothermally. After $t_{w}$, the field is cut to zero, $H_{f c} \rightarrow 0$, and the logarithmically slow TRM time decay is monitored up to the macroscopic times. The TRM decay depends on the aging temperature $T_{m}$, the aging time $t_{w}$ and the field $H_{f c}$. In the following we elaborate these dependencies for the CoCrFeMnNi HEA in detail.

In the first set of experiments (TRM versus $T_{m}$ ), the sample was cooled in a small field $\mu_{0} H_{f c}=0.5 \mathrm{mT}$ from the starting temperature $100 \mathrm{~K}$ to different aging temperatures $T_{m}=25,20,15,10,7.5,4$ and $1.8 \mathrm{~K}$. At $T_{m}$, aging for $t_{w}=1 \mathrm{~h}$ was employed in all cases and the TRM time decay was then monitored for $t \approx 1700 \mathrm{~min}$ (approx. $29 \mathrm{~h}$ ) after the $H_{f c}$ switch-off (which was accomplished in about $10 \mathrm{sec}$ ). The timedecay curves, normalized to the magnetization prior to cutting the field to zero, $M_{T R M}\left(T_{m}, t\right) / M_{f c}\left(T_{m}\right)$ are shown in Fig. 8. It is observed that the magnitude of the TRM strongly increases upon lowering the temperature, the logarithmically slow time decay significantly slows down on lowering the $T_{m}$ and no TRM exists at $T_{m}=25 \mathrm{~K}$ (just above $T_{f} \approx 20$ $\mathrm{K})$, in the ergodic phase. The normalized TRM amplitude at the 

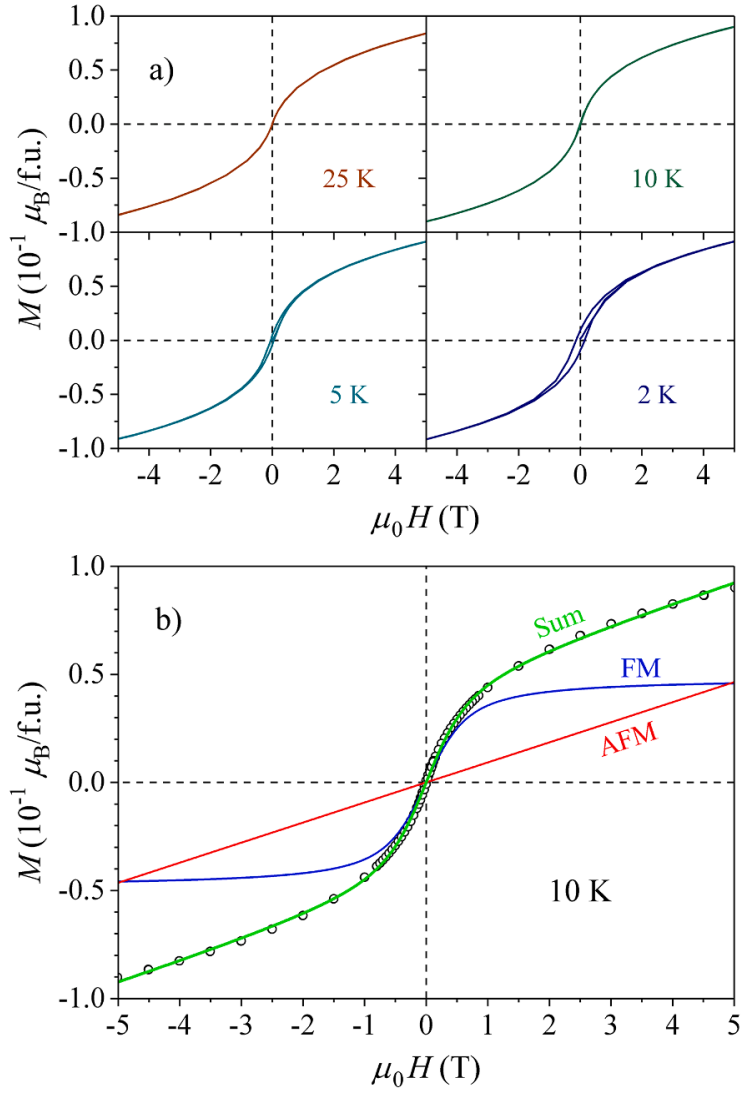

c)

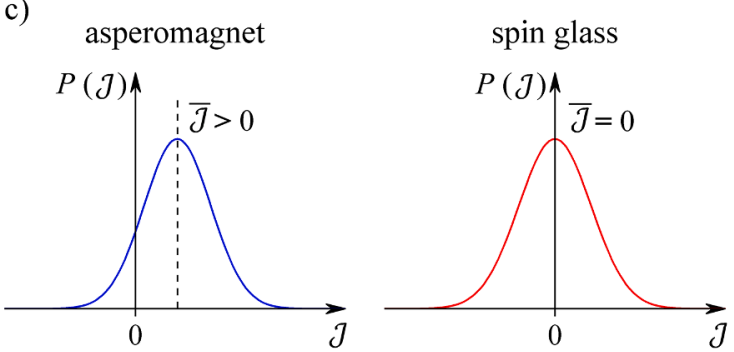

Fig. 7. (a) The magnetization versus the magnetic field curves, $M(H)$, at selected temperatures between 25 and $2 \mathrm{~K}$. (b) The fit of the $M(H)$ curve at $T=$ $10 \mathrm{~K}$ with Eq. (1) (fit parameters are given in the text), where the FM and AFM contributions to the total $M$ are also shown separately. (c) Schematic presentation of the distribution function of the exchange coupling constants $P(\mathscr{f})$ for a spin glass $\left(\mathscr{J}^{-}=0\right)$ and asperomagnet $\left(\mathscr{J}^{-}>0\right)$.

beginning of the decay, as a function of $T_{m}, M_{T R M}\left(T_{m}, t=0\right) / M_{f c}\left(T_{m}\right)$, is shown in the inset of Fig. 8.

In the second set of experiments, the TRM time decays were recorded as a function of the aging time $t_{w}$ at a fixed aging temperature $T_{m}=10$ K. The sample was field-cooled to $T_{m}$ in $\mu_{0} H_{f c}=0.5 \mathrm{mT}$ and was left to age in that field for $t_{w}=5 \mathrm{~min}, 20 \mathrm{~min}, 1 \mathrm{~h}, 2 \mathrm{~h}, 4 \mathrm{~h}$ and $8 \mathrm{~h}$. After turning the field off, $H_{f c} \rightarrow 0$, the TRM time decays were monitored up to $t=180 \mathrm{~min}$. The normalized time-decay curves $M_{\text {TRM }}\left(t_{w}, t\right) / M_{f c}\left(t_{w}\right)$ are shown in Fig. 9. It is evident that the TRM increases with increasing $t_{w}$, the decays slow down and the shape of the decay curves also changes (the remanence of the spin system increases). The last two features are more clearly visible in the inset of Fig. 9, where the TRM decay curves, as a function of $t_{w}$, are shown normalized to their $t=0$ values, $M_{T R M}\left(t_{w}\right.$, $t) / M_{\text {TRM }}\left(t_{w}, t=0\right)$.

In the third set of experiments, the TRM time decays were measured as a function of the field $H_{f c}$. The sample was field-cooled to $T_{m}=10 \mathrm{~K}$ and left there to age for $t_{w} 1 \mathrm{~h}$ before cutting the field to zero. The

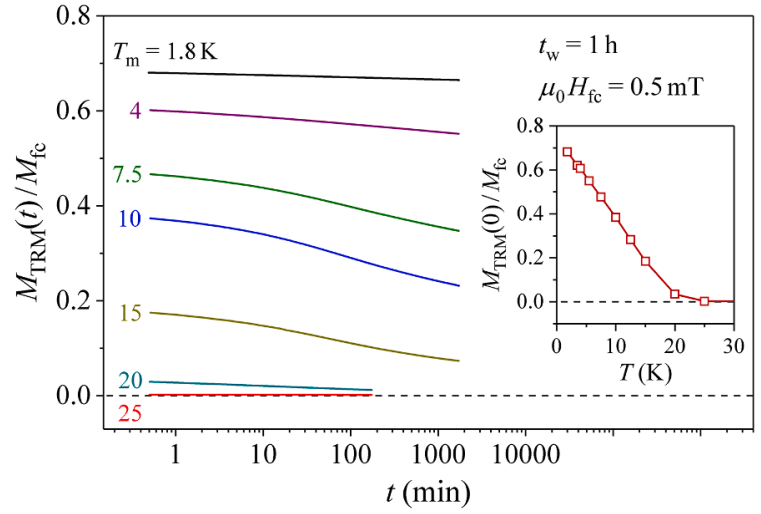

Fig. 8. TRM time-decay curves, normalized to the magnetization prior to cutting the field to zero, $M_{T R M}\left(T_{m}, t\right) / M_{f c}\left(T_{m}\right)$ at different aging temperatures $T_{m}$ between 25 and $1.8 \mathrm{~K}$. The inset shows the normalized TRM amplitude at the beginning of the decay, $M_{T R M}\left(T_{m}, t=0\right) / M_{f c}\left(T_{m}\right)$, as a function of $T_{m}$.

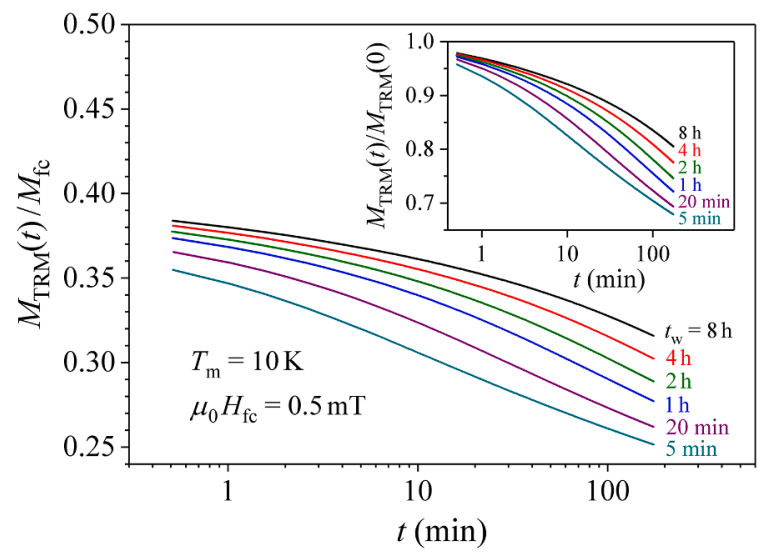

Fig. 9. TRM time-decay curves, normalized to the magnetization prior to cutting the field to zero, $M_{T R M}\left(t_{w}, t\right) / M_{f c}\left(t_{w}\right)$ at $T_{m}=10 \mathrm{~K}$, as a function of the aging time $t_{w}$. The inset shows the TRM decay curves normalized to their $t=$ 0 values, $M_{\text {TRM }}\left(t_{w}, t\right) / M_{T R M}\left(t_{w}, t=0\right)$, as a function of $t_{w}$.

following field values were employed: $\mu_{0} H_{f c}=0.5,5,10,20,50,100$ $\mathrm{mT}$ and $1 \mathrm{~T}$. The normalized TRM time decays, $M_{T R M}\left(H_{f c}, t\right) / M_{f c}\left(H_{f c}\right)$, are displayed in Fig. 10. It is evident that the TRM decreases strongly

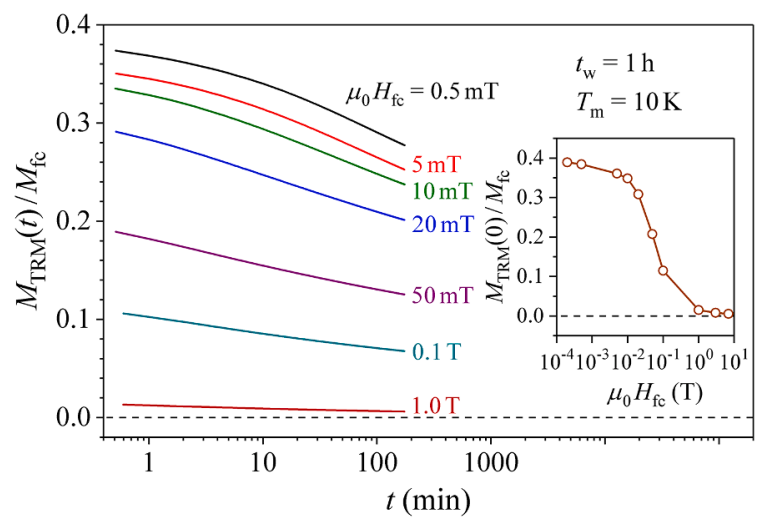

Fig. 10. TRM time-decay curves, normalized to the magnetization prior to cutting the field to zero, $M_{T R M}\left(H_{f c}, t\right) / M_{f c}\left(H_{f c}\right)$, at $T_{m}=10 \mathrm{~K}$, as a function of the aging field $H_{f c}$. The inset shows the TRM $t=0$ amplitude, $M_{T R M}\left(H_{f c}, t=0\right) / M_{f c}\left(H_{f c}\right)$, as a function of $H_{f c}$ (note that the $H_{f c}$ scale is logarithmic). 
with the increasing $H_{f c}$. The TRM amplitude $M_{T R M}\left(H_{f c}, t=0\right) / M_{f c}\left(H_{f c}\right)$, as a function of $H_{f c}$ is displayed in the inset of Fig. 10, where a decrease by a large factor of about 30 in the field range $0.5 \mathrm{mT}<\mu_{0} H_{f c}<1 \mathrm{~T}$ is evident.

\subsection{Memory effect}

The memory effect (ME) is another manifestation of the out-ofequilibrium dynamics of a magnetically frustrated spin system with a broken ergodicity $[20,19,23]$. To observe the ME, the spin system is cooled in zero field from the paramagnetic phase into the nonergodic phase and the cooling is stopped at $T_{m}<T_{f}$ for a time $t_{w}$. After isothermal aging at $T_{m}$ for $t_{w}$ of the order of minutes to hours, the cooling is resumed. At the lowest temperature, a small magnetic field (of a few tenths of a mT) is applied and the zfc magnetization is measured in a continuous heating run. A diminution of $M_{z f c}$ is observed at $T_{m}$ with respect to the $M_{z f c}$ of the no-aging case ( $\left.t_{w}=0\right)$ ), and the magnitude of the diminution depends on the aging temperature $T_{m}$ and the aging time $t_{w}$. The diminution (a dip) in the $M_{z f c}$ at $T_{m}$ represents a "thermal imprint" into the magnetization, which provides the basis of a thermal memory cell [24] for storing a byte of digital information into the material by pure thermal manipulation.

In the first set of experiments (ME versus aging time $t_{w}$ ), the $\mathrm{zfc}$ cooling was performed with the cooling rate of $0.5 \mathrm{~K} / \mathrm{min}$ and was stopped at $T_{m}=10 \mathrm{~K}<T_{f}$, where the spin system was aged isothermally for times $t_{w}=10 \mathrm{~min}, 30 \mathrm{~min}, 1 \mathrm{~h}, 4 \mathrm{~h}$ and $8 \mathrm{~h}$. After $t_{w}$, the cooling has resumed to $2 \mathrm{~K}$. A reference run with no stop $\left(t_{w}=0\right)$ was also performed. At the lowest temperature, a magnetic field $\mu_{0} H_{z f c}=0.5 \mathrm{mT}$ (equivalent to 5 Oe in cgs units) was applied and $M_{z f c}$ was measured in a heating run. A dip in the aged curves $M_{z f c}\left(t_{w} \neq 0\right)$ at $T_{m}$ with respect to the reference (unaged) curve $M_{z f c}\left(t_{w}=0\right.$ ) is clearly observed (Fig. 11a). Fig. 11b shows an expanded portion of the curves in the vicinity of $T_{m}$, where it is seen that the ME is stronger (the dip in the $M_{z f c}$ is deeper) for longer aging times. In Fig. 11c, the normalized (dimensionless) difference between the reference curve and the aged curves, $\Delta M=$ $\left[M_{z f c}\left(t_{w}=0\right)-M_{z f c}\left(t_{w}\right)\right] / M_{z f c}\left(t_{w}=0\right)$, is displayed, where it is seen that the difference for the longest $t_{w}=8 \mathrm{~h}$ amounts to $\Delta M=7 \% . \Delta M$ resembles a resonant curve peaking at the aging temperature $T_{m}=10 \mathrm{~K}$ (with a small undershot on the high-temperature side), being smeared over a finite temperature interval of $\pm 2 \mathrm{~K}$ around $T_{m}$. The frustrated spin system in the CoCrFeMnNi HEA therefore remembers both the temperature and the time of aging.

In the second set of experiments, multiple consecutive stops within the nonergodic phase during the same zfc cooling run were performed, allowing the spin system to age at each stop temperature. Fig. 12a shows the $M_{z f c}$ after three consecutive stops at $T_{m 1}=18 \mathrm{~K}, T_{m 2}=12 \mathrm{~K}$ and
$T_{m 3}=8 \mathrm{~K}$ with the aging for $t_{w}=4 \mathrm{~h}$ at each temperature. The reference $\left(t_{w}=0\right)$ run was also performed. The normalized difference $\Delta M$ is shown in Fig. $12 \mathrm{~b}$, where three well resolved peaks at the selected stop temperatures $T_{m i}$ are clearly seen. All three stops have therefore been memorized by the spin system. In Fig. $12 \mathrm{c}$, the $M_{z f c}$ is shown for the case where the cooling was stopped for $t_{w}=4 \mathrm{~h}$ at seven consecutive temperatures $T_{m i}(i=1-7)$ between 18 and $6 \mathrm{~K}$ in steps of $\Delta T_{m}=2 \mathrm{~K}$. It is evident that all seven stops have been memorized. The seven peaks in $\Delta M$ are clearly recognizable (Fig. 12d), but due to their finite widths, they partially overlap at the baseline.

\section{Discussion}

The TRM ultraslow time decay and the ME are two typical out-ofequilibrium phenomena observed in nonergodic, magnetically frustrated systems. The same type of the $T_{m^{-}}, t_{w^{-}}$and $H_{f c}$-dependence of the TRM and the ME, as observed for the CoCrFeMnNi HEA were reported before for spin glasses $[25,20,21,22]$ and geometrically frustrated quasicrystals and complex metallic alloys $[26,23]$. Being non-equilibrium phenomena, the TRM time decay and the ME are difficult to treat theoretically in a quantitative manner, because the observed physical quantities are time-dependent, defined by the finite time scale of a given experimental observation technique. The TRM and the ME phenomena are currently still incompletely understood and comprehensive literature on the subject exists $[20-23,25,26]$. The explanations of the aging phenomena are based on the specific shape of the free-energy surface in the phase space, which is highly degenerate because of the randomness and frustration of the spin system.

The free-energy landscape exhibits many local and global minima, separated by energy barriers of different heights (shown schematically in Fig. 13) [10,27-29], where each minimum represents a collective metastable spin state. This kind of a free-energy landscape (called ultrametric) was theoretically predicted for spin glasses by the replicasymmetric solution of the Sherrington-Kirkpatrick model [27]. In order to reach thermal equilibrium at a given temperature, the spin system should visit the whole phase space via thermal reorientations of the spins, equivalent to jumping over the barriers in the free-energy phase space (assuming classical over-barrier hopping processes). Below the spin freezing temperature $T_{f}$, the size of the phase space and the wide distribution of the barriers prevent the spin system to reach thermal equilibrium on finite experimental time scales, resulting in a broken ergodicity of the spin system. The spin dynamics can be viewed as a random walk in the phase space and the portion of the space visited depends on the observation time, making the observable physical quantities time-dependent instead of being time-independent thermodynamic quantities. Higher temperatures $T_{m}$ (but still below $T_{f}$ ), longer
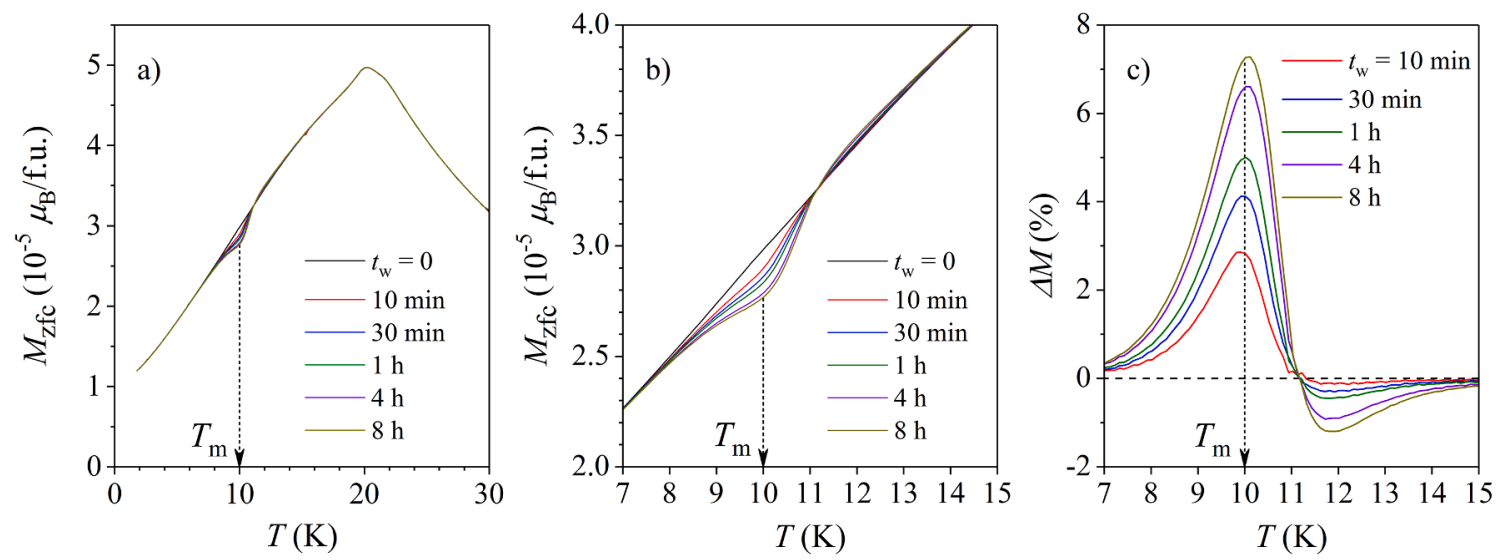

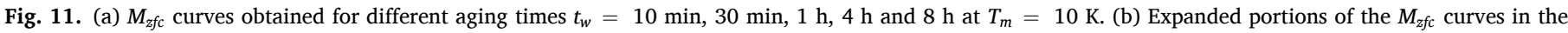
vicinity of $T_{m}$. (c) normalized difference between the reference (unaged) curve $M_{z f c}\left(t_{w}=0\right)$ and the aged curves, $\Delta M=\left[M_{z f c}\left(t_{w}=0\right)-M_{z f c}\left(t_{w}\right)\right] / M_{z f c}\left(t_{w}=0\right)$. 

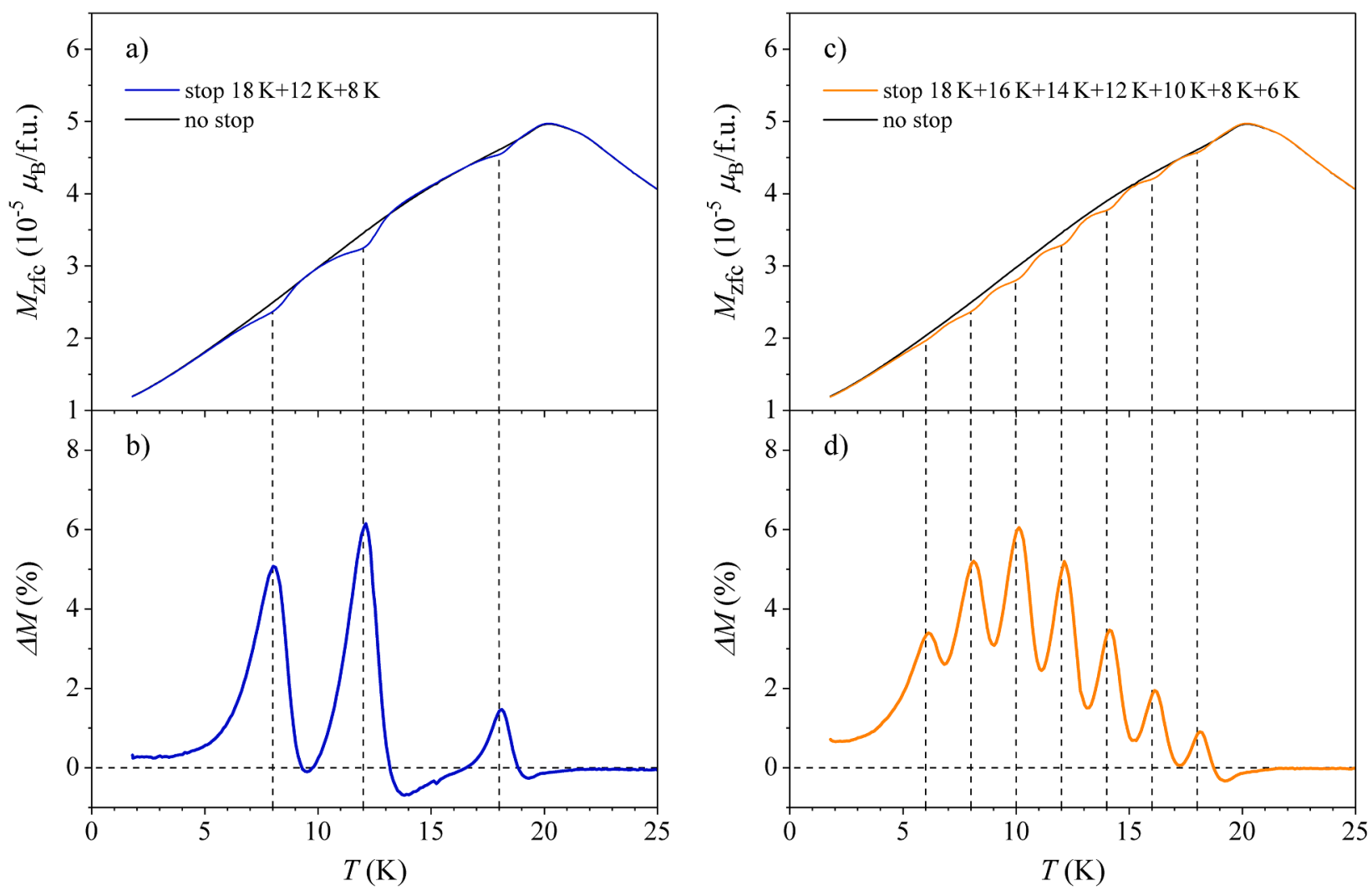

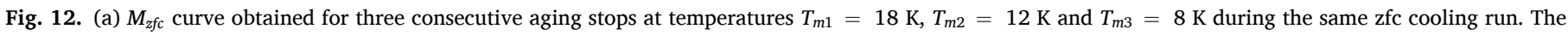

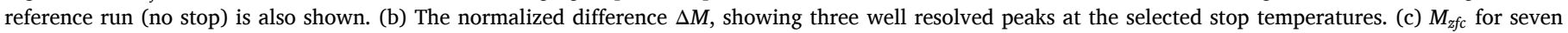
consecutive stops at temperatures $T_{m i}(i=1-7)$ between 18 and $6 \mathrm{~K}$ in steps of $\Delta T_{m}=2 \mathrm{~K}$. (d) The normalized difference $\Delta M$ for the seven stops.

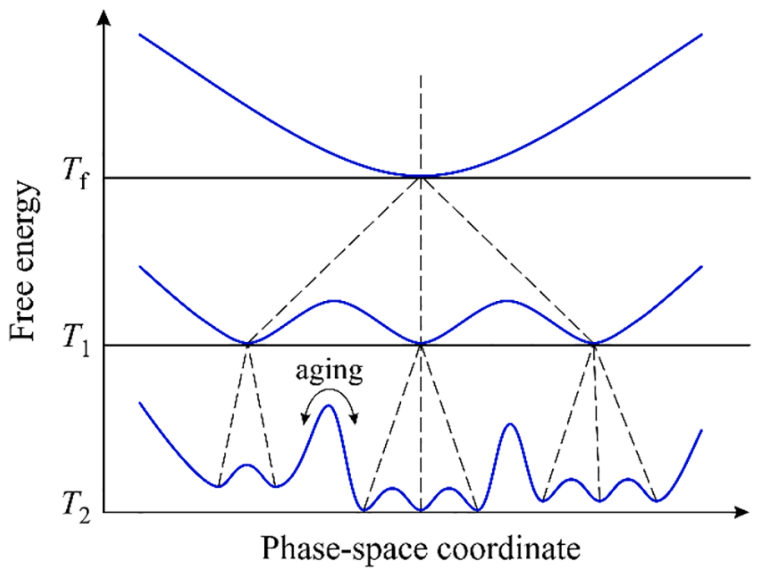

Fig. 13. Free-energy surface for the ultrametric organization of metastable states in spin glasses $[27,29]$. When the temperature is lowered $\left(T_{2}<T_{1}<T_{f}\right)$, each valley subdivides into others. The barriers between valleys "born" from the same ancestor state are small, whereas they are increasingly higher for states that have the closest common ancestor higher on the hierarchical tree (shown by the dashed lines). Aging denotes jumping of the system over potential barriers during the waiting (aging) time $t_{w}$.

aging times $t_{w}$ and smaller magnetic fields $H_{f c}$ enable the spin system to visit a larger part of the phase space, resulting in the $T_{m^{-}}, t_{w^{-}}$and $H_{f c}$-dependences of the TRM and the ME as presented in Figs. 8-12. For further details, the reader is referred to the literature $[20-23,25,26]$. The essential conclusion here is that the CoCrFeMnNi HEA exhibits identical out-of-equilibrium spin dynamics as other magnetically frustrated systems (electrically conducting and insulating spin glasses [19,20,30,31], geometrically frustrated complex metallic alloys [23], magnetic quasicrystals [26] and magnetic nanoparticles [32,33]). This kind of out-ofequilibrium dynamics is not restricted to the magnetically frustrated systems only, but is also characteristic of electrically frustrated systems like disordered ferroelectrics [34,35] and orientational glasses [36-38].

\section{Conclusions}

The CoCrFeMnNi HEA is a magnetically concentrated crystalline system with all lattice sites magnetic, containing randomness (five different types of spins are randomly positioned in the lattice) and frustration (a consequence of mixed FM/AFM interactions), which allow classifying this HEA into the broad class of spin glasses. Upon cooling, no long-range magnetic ordering (FM or AFM) takes place, but the spin system undergoes a kinetic spin freezing transition, where below the spin freezing temperature $T_{f} \approx 20 \mathrm{~K}$, the ergodicity of the spin system is broken on the experimental time scale. The broken-ergodicity phenomena observed below $T_{f}$ include zfc-fc magnetization splitting in low magnetic fields, a frequency-dependent cusp in the ac susceptibility, hysteresis and remanence, an ultraslow time-decay of the thermoremanent magnetization and the memory effect. All these phenomena are associated with the out-of-equilibrium dynamics of a nonergodic, frustrated system of coupled spins that cannot reach thermal equilibrium on a finite experimental time scale. At any temperature within the nonergodic phase, the spin system evolves towards a global equilibrium, but since the equilibration requires macroscopic times, we observe experimentally only transient effects of a partial equilibration within localized spin domains. The degree of quasi-equilibration depends on the aging time spent at a given temperature under steady external conditions. Such non-equilibrium dynamics is difficult to treat theoretically, as the observable physical quantities are time-dependent. The spin glass behavior of the CoCrFeMnNi HEA is in agreement with the recent report 
on this HEA [6], where only slightly higher spin freezing temperature of $T_{f} \approx 25 \mathrm{~K}$ was found from the temperature-dependent fc magnetization (very likely due to slightly different chemical composition).

\section{Experimental section}

The sample material was grown by the Czochralski technique. An ingot of high-purity elements $\mathrm{Fe}, \mathrm{Co}, \mathrm{Cr}, \mathrm{Mn}$, and $\mathrm{Ni}$ was pre-alloyed by high-frequency levitation melting in a cold copper crucible with several remelting cycles to achieve a high homogeneity. Czochralski growth was carried out under 800 mbar argon pressure from a $\mathrm{ZrO}$ crucible placed in a tungsten susceptor for inductively coupled high-frequency heating. We used a polycrystalline rod of $2 \mathrm{~mm}$ diameter for homogeneous seeding. The pulling speed was initially set to $10 \mathrm{~mm} / \mathrm{h}$ and lowered to $1 \mathrm{~mm} / \mathrm{h}$ after the final desired crystal diameter of about $1 \mathrm{~cm}$ was reached. The growth was terminated after the crystal had a length of about $3 \mathrm{~cm}$. The resulting crystal had a clean and shiny surface. From the crystal, samples for further investigation were cut by spark erosion.

Atom probe tomography measurements were performed by a CAMECA LEAP 4000X-HR instrument. The specimens for the APT analyses were fabricated using lift out and FIB milling with Xe ions in a plasma FIB-SEM system (Thermo Fisher Helios G4 PFIB CXe). The tips were conically tapered with a tip radius of around $50 \mathrm{~nm}$ or less. The APT tips were then subjected to voltage pulsing with $20 \%$ pulse to standing ratio at $200 \mathrm{kHz}$ at a base temperature of $50 \mathrm{~K}$, and a detection rate of $0.5 \mathrm{ion} / \mathrm{pulse}$. Data were processed using IVAS 3.8.0 visualization software (CAMECA). All ions were collected in the doubly charged state, with marginal contribution of Ni singly charged. Mass resolution (m/ $\Delta \mathrm{m}$ ) was about 1000 , allowing unambiguous identification of all species.

HAADF STEM imaging and EDS elemental mapping were performed by using a probe Cs-corrected Jeol ARM 200 CF STEM equipped with an SDD Jeol Centurio energy-dispersive X-ray spectrometer. The operating voltage was set to $200 \mathrm{kV}$. Gatan Quantum ER Dual EELS system was used for the EELS spectra collection and sample thickness estimation. The sample for STEM analysis was prepared using a tripod polishing method (Multiprep polishing system from Allied), where the sample was first mechanically thinned to a few micrometers and finally thinned down to electron transparency using a Gatan PIPS II ion-milling system. The final sample thickness in the analyzed areas was about $10 \mathrm{~nm}$.

Magnetic measurements were conducted in part on a QD MPMS3 magnetometer and in part on a QD MPMS XL-5 magnetometer, both operating at temperatures between 1.9 and $400 \mathrm{~K}$. The thermoremanent magnetization at the lowest magnetic field of $0.5 \mathrm{mT}$ and all the memory effect experiments were conducted using a copper AC/ULF coil of the MPMS3 magnetometer to ensure an accurate and repeatable magnetic field. Prior to the low-field experiments, the Ultra Low Field option was used to compensate the residual magnetic field of the superconducting magnet. A bar-shaped sample of dimensions $7 \times 2 \times 2 \mathrm{~mm}^{3}$ was used, with the long axis parallel to the magnetic field.

\section{CRediT authorship contribution statement}

P. Koželj: Investigation, Visualization, Validation, Formal analysis. S. Vrtnik: Investigation, Visualization, Validation, Formal analysis. M. Krnel: Investigation, Visualization, Validation, Formal analysis. A. Jelen: Investigation, Visualization, Validation, Formal analysis. D. Gačnik: . M. Wencka: Investigation, Visualization, Validation, Formal analysis. Z. Jagličić: . A. Meden: Investigation, Visualization, Validation, Formal analysis. G. Dražić: . F. Danoix: Investigation, Visualization, Validation, Formal analysis. J. Ledieu: Investigation, Visualization, Validation, Formal analysis. M. Feuerbacher: Conceptualization, Resources, Writing - review \& editing. J. Dolinšek: Conceptualization, Supervision, Writing - original draft.

\section{Declaration of Competing Interest}

The authors declare that they have no known competing financial interests or personal relationships that could have appeared to influence the work reported in this paper.

\section{Acknowledgments}

The Slovenian authors acknowledge the financial support from the Slovenian Research Agency (research core funding No. P1-0125) and thank the CENN Nanocenter for the use of FIB HeliosNanolab instrument. MF acknowledges financial support from the German Research foundation (DFG) under grant No. FE 571/4 within the priority program SPP2006 "Compositionally Complex Alloys - High Entropy Alloys (CCAHEA)". APT work was carried out owing to experimental GENESIS platform. GENESIS is supported by the Région Haute-Normandie, the Métropole Rouen Normandie, the CNRS via LABEX EMC, and the French National Research Agency as a part of the program "Investissements d'avenir" with the reference ANR-11-EQPX-0020. This work is a result of cooperation within the European C-MAC and within the FrenchSlovene collaboration established under Push-Pull Alloys and Complex Compounds (PACS2) Joint Open Laboratory.

\section{References}

[1] O. Schneeweiss, M. Friák, M. Dudová, D. Holec, M. Šob, D. Kriegner, V. Holý, P. Beran, E.P. George, J. Neugebauer, A. Dlouhý, Phys. Rev. B 96 (2017), 014437.

[2] Y. Zhang, T. Zuo, Y. Cheng, P.K. Liaw, Sci. Rep. 3 (2013) 1455.

[3] F. Tian, L.K. Varga, N. Chen, L. Delczeg, L. Vitos, Phys. Rev. B 87 (2013), 075144.

[4] D. Ma, B. Grabowski, F. Koermann, J. Neugebauer, D. Raabe, Acta Mater. 100 (2015) 90.

[5] Z. Dong, S. Schönecker, W. Li, D. Chen, L. Vitos, Sci. Rep. 8 (2018) 12211.

[6] K. Jin, B.C. Sales, G.M. Stocks, G.D. Samolyuk, M. Daene, W.J. Weber, Y. Zhang, H. Bei, Sci. Rep. 6 (2016) 20159.

[7] B. Schuh, F. Mendez-Martin, B. Voelker, E.P. George, H. Clemens, R. Pippan, A. Hohenwarter, Acta Mater. 96 (2015) 258.

[8] E.J. Pickering, R. Munoz-Moreno, H.J. Stone, N.G. Jones, Scr. Mater. 113 (2016) 106.

[9] F. Otto, A. Dlouhy, K.G. Pradeep, M. Kubenova, D. Raabe, G. Eggeler, E.P. George, Acta Mater. 112 (2016) 40.

[10] K. Binder, A.P. Young, Rev. Mod. Phys. 58 (1986) 801, and references therein.

[11] T.F. Kelly, Atom probe tomography, in: P.W. Hawkes, J.C.H. Spence (Eds.), Springer Handbook of Microscopy, Springer Nature Switzerland, 2019, pp. 715-763. DOI: 10.1007/978-3-030-00069-1.

[12] F.E. Mabbs, D.J. Machin, Magnetism and Transition Metal Complexes, Chapman and Hall, London, 1973, p. 7.

[13] J.A. Mydosh, Spin glasses: an experimental introduction, Taylor \& Francis, London, 1993, p. 67.

[14] J.M.D. Coey, Magnetism and Magnetic Materials, Cambridge University Press, Cambridge, 2010, pp. 209-218.

[15] R.C. O'Handley, Modern Magnetic Materials: Principles and Applications, Wiley, New York, 1999, pp. 391-410.

[16] J.-P. Bouchaud, L.F. Cugliandolo, J. Kurchan, M. Mézard, in: Spin Glasses and Random Fields, World Scientific Singapore, 1998, pp. 161-224.

[17] N. Kawashima, H. Rieger, in Frustrated Spin Systems, ed. H.T. Diep (World Scientific Singapore, 2004), pp. 491-586.

[18] E. Vincent, J. Hammann, M. Ocio, J.-P. Bouchaud, L.F. Cugliandolo, in Complex Behaviour of Glassy Systems, Lecture Notes in Physics 492, ed. M. Rubi (SpringerVerlag, Berlin, 1997), pp. 184-219.

[19] P. Nordblad, P. Svedlindh, in: Spin Glasses Random Fields, World Scientific, Singapore, 1998, pp. 1-28.

[20] J.-P. Bouchaud, V. Dupuis, J. Hammann, E. Vincent, Phys. Rev. B 65 (2001), 024439.

[21] M. Lederman, R. Orbach, J.M. Hammann, M. Ocio, E. Vincent, Phys. Rev. B 44 (1991) 7403.

[22] D. Chu, G.G. Kenning, R. Orbach, Phil. Mag. B 71 (1995) 479.

[23] J. Dolinšek, J. Slanovec, Z. Jagličić, M. Heggen, S. Balanetskyy, M. Feuerbacher, K. Urban, Phys. Rev. B 77 (2008), 064430.

[24] J. Dolinšek, M. Feuerbacher, M. Jagodič, Z. Jagličić, M. Heggen, K. Urban, J. Appl. Phys. 106 (2009), 043917.

[25] Ph. Refregier, E. Vincent, J. Hammann, M. Ocio, J. Phys. (Paris) 48 (1987) 1533.

[26] J. Dolinšek, Z. Jagličić, T.J. Sato, J.Q. Guo, A.P. Tsai, J. Phys.: Condens. Matter 15 (2003) 7981.

[27] G. Parisi, Phys. Rev. Lett. 50 (1983) 1946.

[28] M. Mezard, G. Parisi, N. Sourlas, G. Toulouse, M.A. Virasoro, J. Phys. (Paris) 45 (1984) 843.

[29] V.S. Dotsenko, J. Phys. C 18 (1985) 6023. 
[30] K. Jonason, E. Vincent, J. Hammann, J.P. Bouchaud, P. Nordblad, Phys. Rev. Lett. 81 (1998) 3243

[31] V. Dupuis, E. Vincent, J.P. Bouchaud, J. Hammann, A. Ito, H. Aruga Katori, Phys. Rev. B 64 (2001), 174204.

[32] P. Jönsson, M.F. Hansen, P. Nordblad, Phys. Rev, B 61 (2000) 1261.

[33] O. Cador, F. Grasset, H. Haneda, J. Etourneau, J. Magn. Magn. Mater. 268 (2004) 232.
[34] P. Doussineau, T. Lacerda-Aroso, A. Levelut, Europhys. Lett. 46 (1999) 401.

[35] E.V. Colla, L.K. Chao, M.B. Weissman, D.D. Viehland, Phys. Rev. Lett. 85 (2000) 3033.

[36] A.V. Kityk, M.C. Rheinstädter, K. Knorr, H. Rieger, Phys. Rev. B 65 (2002), 144415.

[37] F. Alberici-Kious, J.P. Bouchaud, L.F. Cugliandolo, P. Doussineau, A. Levelut, Phys. Rev. Lett. 81 (1998) 4987.

[38] F. Alberici, P. Doussineau, A. Levelut, Europhys. Lett. 39 (1997) 329. 